\title{
Geografía del delito. Un estudio de caso ${ }^{1}$
}

Marina de la Torre ${ }^{2}$

\section{Resumen}

El espacio público de nuestras ciudades está fuertemente impactado por las acciones defensivas de sus habitantes frente a los embates de la violencia urbana y su consecuencia natural: la sensación de inseguridad. ¿Cómo se distribuye la violencia en el territorio de la ciudad? ¿Cuáles son los patrones espaciales de comportamiento dominantes? ¿Cuáles los aspectos situacionales que la promueven? Para responder a estas interrogantes, nos proponemos explorar la distribución espacial de la ocurrencia delictiva en la ciudad de León, Guanajuato, durante el año 2010.

Si bien los resultados obtenidos sorprenden por su valor diagnóstico, somos conscientes de los elevados índices de cifra negra (80\% para la ciudad de León), así como del carácter volátil del fenómeno abordado. En tal sentido, estudios precedentes que han obtenido información complementaria a través de la aplicación de Cuestionarios de Victimización, necesitan ser contrastados con el presente diagnóstico geográfico.

Palabras Clave: delito, geografía, patrón de comportamiento.

\section{Abstract}

The public area in our cities is strongly impacted by the defensive actions of its inhabitants against the attacks of urban violence and its most natural consequence: the feeling of insecurity. How violence is distributed in the territory of the city? What are the local patterns of dominant behavior? Which are the situational aspects that encourage it? To answer these questions, we intend to explore the locational distribution of the occurrence of crime in the city of Leon, Guanajuato, during 2010.

1 Se agradece la colaboración de la Universidad De La Salle

2 Doctora en Arquitectura, Universidad de Guanajuato, México. E mail: marinadlt8@hotmail.com 
But while the results are surprising in their diagnostic value, we are aware of the high rates of black figures ( $80 \%$ for the city of Leon), as well as the volatile nature of the phenomenon addressed. In this regard, previous studies have complementary information obtained through the application of Victimization Questionnaires, which need to be contrasted with the present geographic diagnosis.

Keywords: crime, geography, behavior pattern.

\section{INTRODUCCIÓN}

Una de las situaciones más evidentes en relación a la violencia delictiva en América Latina, es el contraste entre la creciente sensación de inseguridad de la población y la ausencia de estadísticas consolidadas que permitan dimensionar de manera más objetiva el fenómeno. Esta situación dificulta la elaboración de diagnósticos que orienten de manera efectiva las acciones a emprender por parte de las autoridades públicas, el sector privado y la población.

Hay avances en el registro estadístico en algunos países - Brasil, Chile, Colombia, El Salvador, Nicaragua, Perú y Venezuela- respecto a la tipificación y niveles de violencia. También lo hay en la medición de los costos económicos y otras formas de evaluación del fenómeno -como las encuestas de victimización- que permiten un análisis de la prevalencia efectiva de la violencia.

\section{Violencia urbana y victimización}

La violencia urbana está asociada, por algunos autores, al concepto de criminalidad (Carrión, 1994; Vanderschueren, 1994). La mayor parte de la violencia ejercida en contra de una persona tiene una dimensión delictiva $y$, por tanto, está penada socialmente. Sin embargo, se adopta el término de violencia urbana para hacer referencia al crimen cometido en los entornos públicos de las grandes ciudades. Así, la violencia urbana sería aquella ejercida en el marco de las relaciones y dinámicas mediadas por la convivencia urbana, cuyas expresiones más frecuentes son el robo a mano armada, las amenazas, las agresiones, los golpes, los secuestros y el homicidio.

La victimización es el acto a través del cual una persona es objeto del uso de la fuerza, cuya consecuencia es el daño físico y/o psicológico. La mayor parte de los estudios revelan que la victimización por violencia urbana, sobre todo la que 
termina con la muerte o con el daño físico de la persona, se encuentra asociada con ciertas variables demográficas.

Las dos variables importantes asociadas con la victimización son las demográficas y las conductuales.

La pertenencia a un sector socioeconómico bajo suele considerarse un factor de riesgo de victimización violenta. Sin embargo, algunos estudios sugieren que la condición personal de la víctima (género y edad) pesa más que el contexto ambiental donde el acto violento se produce. Rose-Ackerman (1997), concluye acerca de un estudio en la ciudad de San Pablo que los robos (delitos contra la propiedad) predominan en áreas donde son mejores las condiciones de vida y, en cambio, los homicidios (delitos contra las personas) ocurren en áreas periféricas de baja extracción económica.

En relación a la variable conductual, el consumo de alcohol y la tenencia de armas parecen estar asociadas con la victimización. Su presencia se convierte en un factor de riesgo de sufrir una agresión. Por lo general, estas variables se han asociado más con el perfil del delincuente que con la víctima.

Cabe distinguir entre los delitos por inseguridad y aquellos por convivencia. Nos dedicaremos a los de inseguridad, es decir, aquellos que suceden en los espacios públicos, pero también en los espacios privados, a mano de terceros.

Principales Sistemas de Sintematización de la Información relacionados con el tema Los primeros antecedentes vinculados a la distribución espacial del delito se remontan a principios del siglo XIX, con la Escuela Cartográfica de Criminología en Francia y posteriormente, en Inglaterra. Este enfoque sociológico asocia directamente las conductas delictivas al crecimiento urbano.

En los años setenta, el arquitecto Oscar Newman (1972), desarrolló un programa de reducción y prevención del crimen basado en el concepto de "espacio defendible", el cual nos refiere al control social de los espacios en la prevención del delito.

Las vinculaciones de la ocurrencia del delito con los Sistemas de Información Geográfica (GIS) se remontan a mediados de los años sesenta. En esta época los sistemas informáticos ofrecían serias limitaciones para el manejo de grandes bases de datos, como así también, dificultades para relacionar múltiples variables. Los primeros "mapas del crimen" se desarrollaron en la Universidad de Harvard. Durante los años ochenta, la convergencia de intereses científicos y académicos, por un lado, con intereses políticos y administrativos, por el otro, facilitaron el impulso ostensible del uso de los GIS en Estados Unidos, Canadá y Reino Unido. 
A principios de los años noventa, la mayor accesibilidad -en precio y simplificación de uso- a estos sistemas permitió su uso por parte de las fuerzas policiales.

En México, en el marco de la Conferencia Nacional de procuración de Justicia, celebrada en marzo de 2007, se dispondría del Sistema de Información Geográfica Geo-referenciada (IGEO), cuyas acciones específicas permitirían: a) establecer enlaces entre las procuradurías estatales y la Nacional, a efectos de homologar criterios para la codificación y registro de delitos; y b) alcanzar la geo-referenciación del delito en la ciudad capital o aquellas de mayor incidencia delictual, en cada uno de los Estados. A nivel nacional, la adopción de este sistema se encuentra en ciernes y no parece haber avances significativos en su aplicación a nivel estatal.

En tal sentido, las exploraciones previas a la propuesta de esta investigación indican que hasta el momento, la ciudad de León no cuenta con una sistematización de datos homologada que permita su georeferenciación.

\section{Software de aplicación}

Los Sistemas de Información Geográfica (GIS) permiten obtener una mejor comprensión de la extensión y distribución del delito dentro de un territorio, en este caso la ciudad de León. Estos sistemas permiten localizar espacialmente, en mapas georeferenciados, el lugar exacto de comisión del hecho delictivo. A partir de este recurso se puede tener una visión multidimensional de los hechos, al poder vincularlos con otras variables de contexto que pueden ser más o menos determinantes para la comisión del hecho delictivo.

En tal sentido, la información del Instituto Nacional de Estadística y Geografía (INEGI) es de extrema utilidad, ya que permite contar con datos acerca de la distribución espacial, volumen y características de la población de los distintos sectores del área a estudiar; además de la localización de elementos significativos de la estructura urbana referidos a educación, salud, seguridad, espacios verdes, etc., todas ellas, identidades georeferenciadas.

\section{ASPECTOS OPERATIVOS}

Esta investigación aplicada combina los enfoques cuantitativo y cualitativo, y su alcance es exploratorio. La selección de indicadores está orientada a la descripción y cualificación del territorio urbano y su relación con el comportamiento delictivo.

En sentido operativo, el abordaje al problema se inicia a partir de las base de datos obtenidas de las agencias de la Procuraduría de Justicia Regional, León, en base a 
las denuncias de delitos registradas. Cada Agencia aporta los registros de un determinado tipo de delito y en conformidad con cada uno de ellos se integra una Base de Datos general. A través del uso de herramientas propias de los Sistemas de Información Geográfica (SIG), se procede a la georeferenciación de los datos obtenidos. Enseguida se procede a elaborar un conjunto de mapas temáticos, en correspondencia con cada una de los indicadores en consideración: a) tipologías delictivas; b) frecuencia de la comisión delictiva; c) lugares vulnerables a la delincuencia; d) zonas calientes, entre otros. Este recurso, el SIG, permite obtener una visión multidimensional de los hechos, al vincularlos con otras variables de contexto que pueden ser más o menos determinantes en la comisión del hecho delictivo.

Las bases de datos aportadas por la Procuraduría, a través de sus numerosas Agencias (más de veinte), comprenden un volumen de aproximadamente 15.000 registros de denuncias. Cada agencia posee su propio formato de registros, situación que dificultó el procesamiento de la información. En tal sentido, hubo necesidad de homologar los criterios de registros para integrarlos en una Base de Datos General, antes de proceder a su georeferenciación (ver tabla T1).

Tabla T1 - Base de Datos homologada

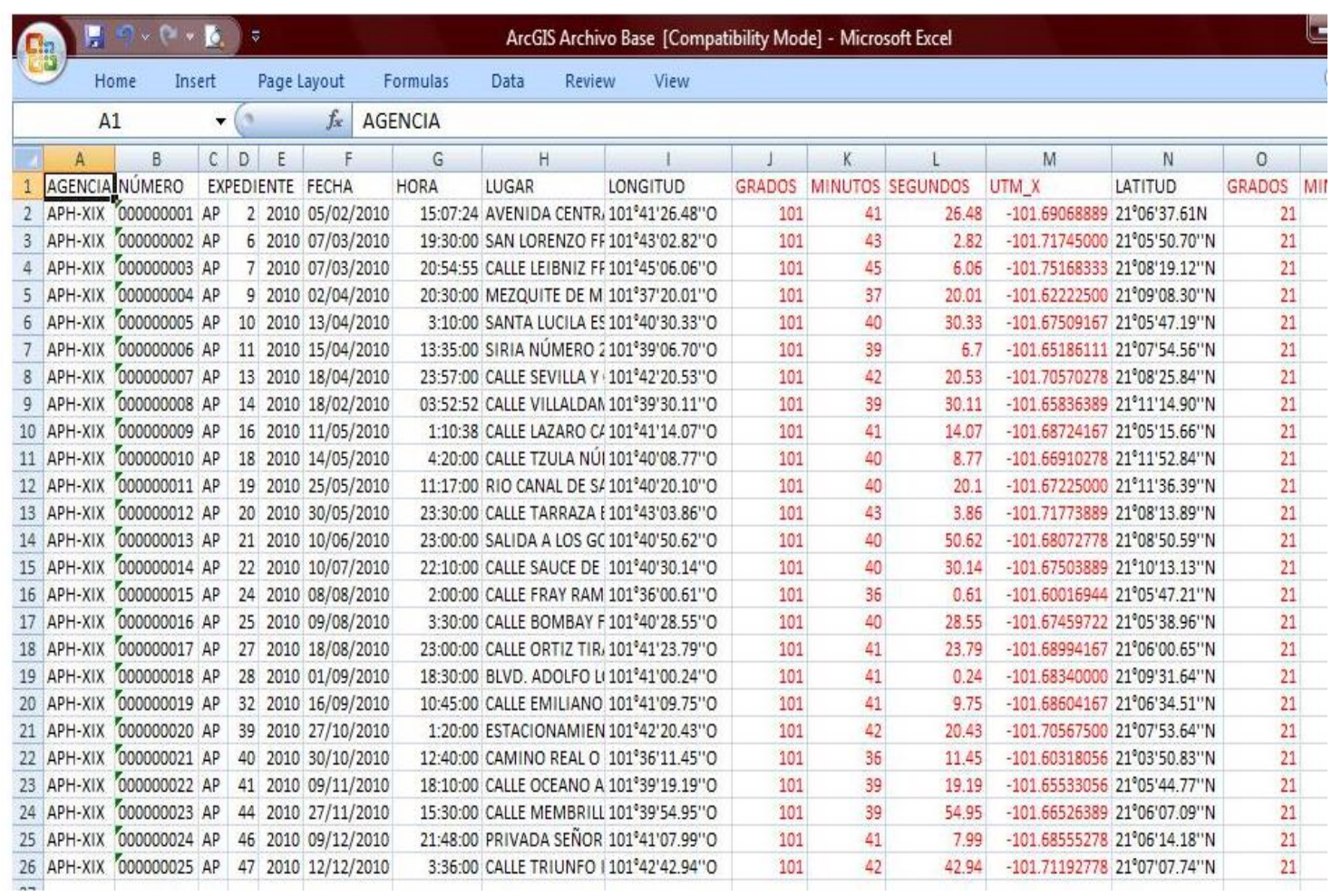


Fuente: elaboración propia, a partir de las bases de datos oficiales proporcionadas por las Agencias de la Procuraduría de Justicia Regional, León.

Por medio de Google Earth, se procedió a georeferenciar los datos obtenidos, a partir del sistema de coordenadas terrestres: altitud y latitud. Estos datos son exportados al ArcGis, el cual permite identificar la localización exacta de cada hecho delictivo en el territorio de la ciudad. En la figura F1 es posible observar la distribución puntual de los delitos cometidos ubicados en el perfil de la planta urbana de la ciudad de León.

Figura F1 - Distribución espacial del conjunto de delitos, ciudad de León

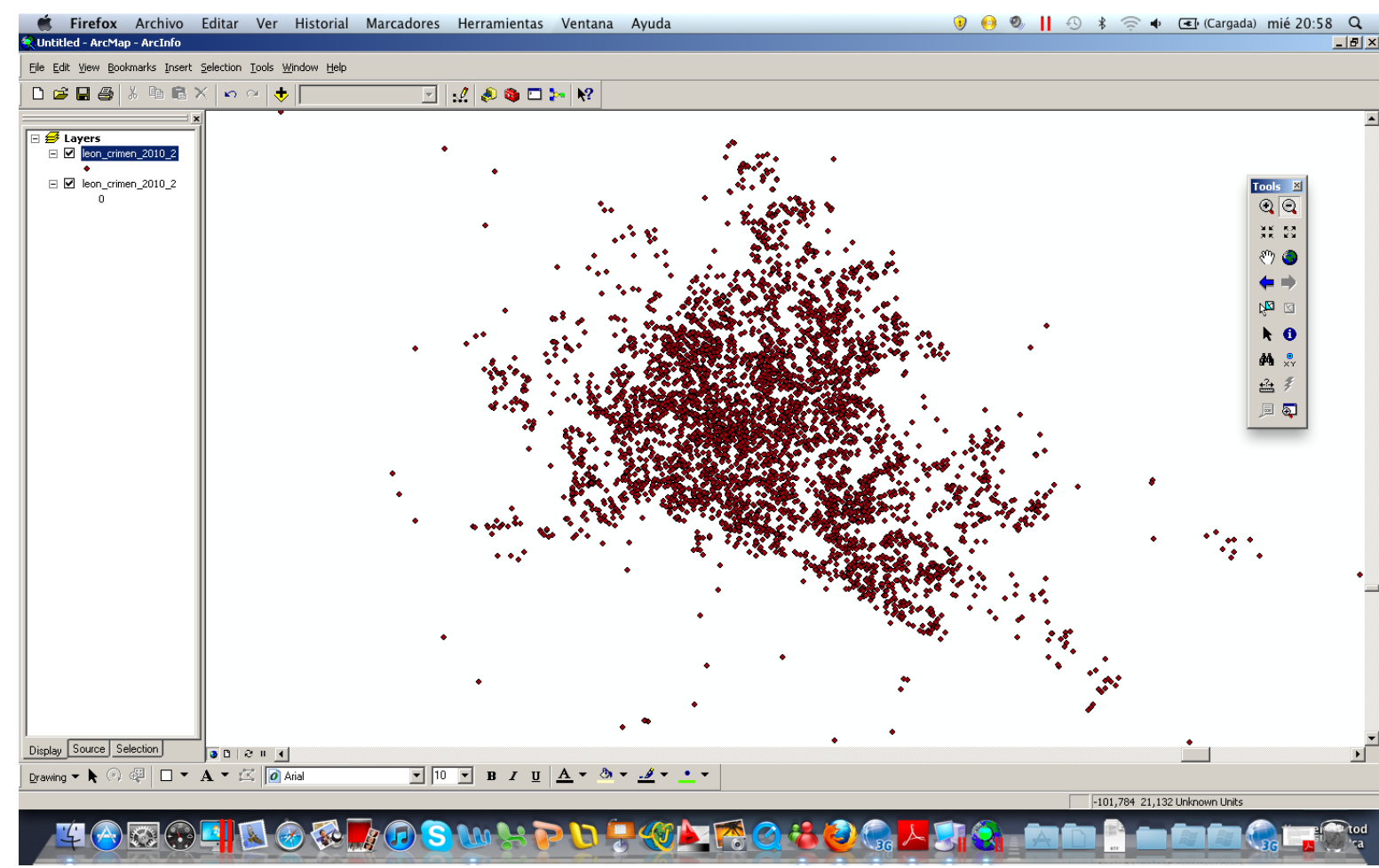

Fuente: elaboración propia, a partir de bases de datos oficiales y su correspondiente georeferenciación a través de aplicación de Google Earth.

Sobre la base de las cartografías digitales del área de estudio, soporte mínimo indispensable para cualquier desarrollo de un SIG, se procede a la elaboración de los mapas temáticos en correspondencia con cada uno de los indicadores propuestos. 


\section{ÁREAS CRÍTICAS}

Se consideran áreas críticas aquellas zonas geográficas donde se concentran altos niveles de intensidad de delitos. Conocer estas localizaciones arroja un aporte significativo a los responsables de la seguridad, investigadores o fuerzas policiales, ya que permite orientar las estrategias de acción y la distribución de los recursos disponibles.

Estas áreas se identifican mediante el cálculo de la densidad de delitos cometidos, 0 los niveles de concentración de los mismos en puntos específicos de la ciudad. Visualizar cómo se distribuyen en el espacio estas áreas y puntos debe ser consistente con el tipo de delito analizado y con la unidad de análisis adoptada. Cuando los delitos están georeferenciados, es preferible analizar los niveles de concentración y por lo tanto trabajar con mapas de puntos.

A las concentraciones de delitos en el espacio se las conoce como puntos calientes. La escala de trabajo modifica el término punto por área, según sea la extensión espacial de la concentración.

Un punto caliente es un área en la que tiene lugar el mayor porcentaje de delitos o es un área en la cual la población tiene el más alto riesgo de victimización.

El Mapa de Densidad delictiva M1 es concluyente: el Centro Histórico de la ciudad de León representa la zona crítica más extendida del municipio.

Mapa M1 - Densidad delictiva, ciudad de León: zonas críticas 


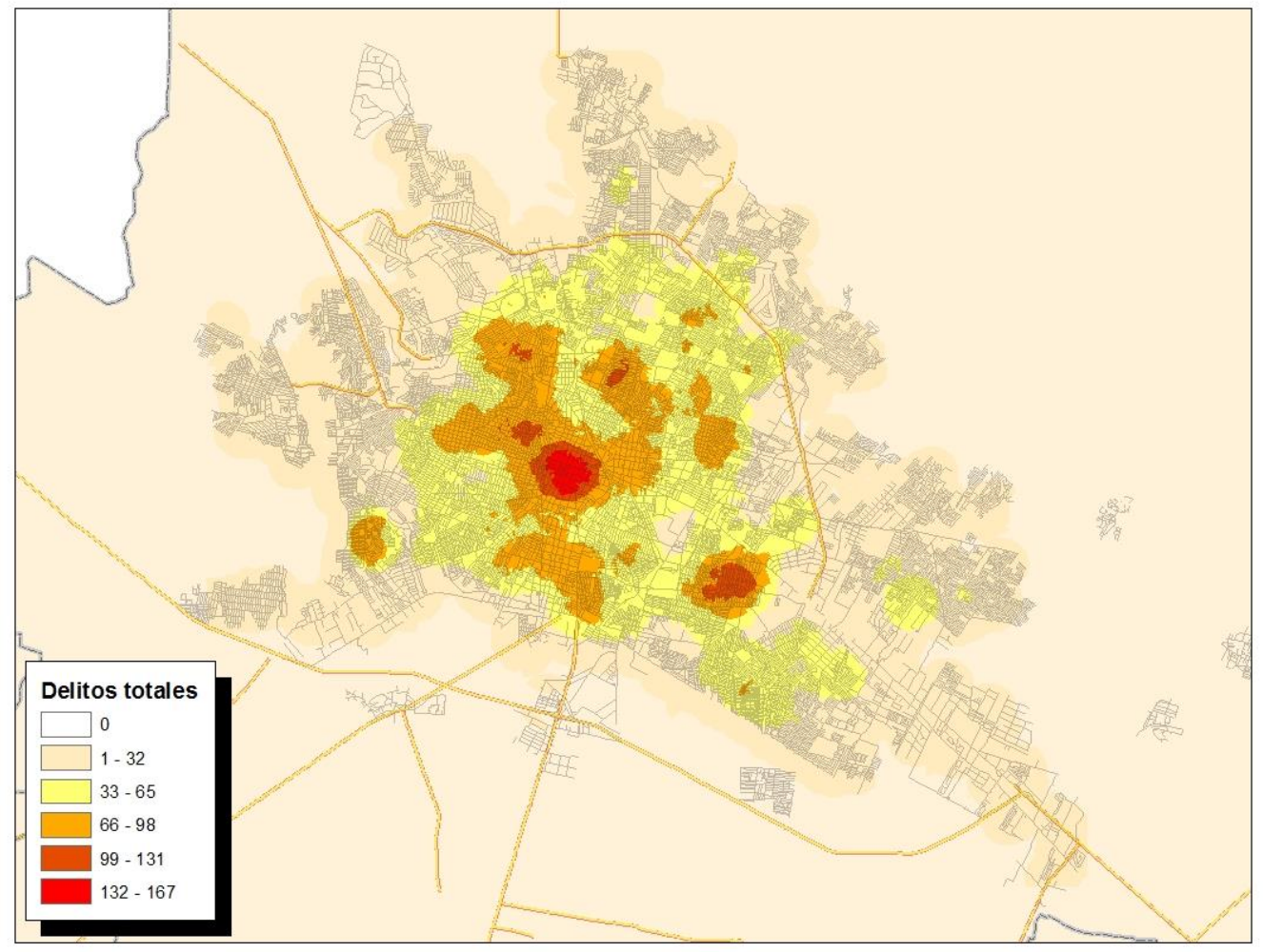

Fuente: elaboración del Ing. Marco Antonio Lira (IPLANEG), a partir de bases de datos oficiales.

\section{Centro Histórico de León}

"El discurso del riesgo empieza donde la confianza en nuestra seguridad termina...", expresa Ulrich Beck. La crisis de la modernidad -caracterizada por la búsqueda de dominio y de control, ha transformado las relaciones entre la Sociedad y el Estado, y entre la Sociedad y la Naturaleza (Beck, 1992).

Sobre la base conceptual de la sociedad en riesgo postulada por Beck, Francois Asher enuncia la idea de la ciudad de los riesgos (Ascher, 2004). Si bien la ciudad es el lugar de las oportunidades y las libertades, a cambio, es también "...el lugar de los peligros físicos y morales...", expresa el autor. Destaca así el carácter ambivalente de las ciudades, cuya doble naturaleza ofrece protección, a la vez que suscita todo tipo de peligros.

Jane Jacobs, una importante defensora de los espacios públicos, argumenta a favor del control y la vigilancia en dichos espacios para que éstos puedan ser apropiadamente disfrutados. La presencia de personas en las calles activa de manera espontánea los controles sociales informales (Jacob, 1961). Sin embargo, la 
presencia de personas en las calles implica la potencial presencia de más víctimas y en consecuencia, más victimarios. Esta realidad nos enfrenta nuevamente al carácter ambivalente de los espacios públicos, es decir, la ciudad por antonomasia.

En tal sentido, proponemos que más que un problema cuantitativo, pareciera tratarse de la calidad de los controles sociales informales. La efectividad y el alcance de dichos controles dependen de los agentes que detentan el control. Si el control social informal es ejercido por el transeúnte ocasional, que va de paso hacia otros destinos, las garantías de seguridad están restringidas a los tiempos de su permanencia en las calles. Si el control social es ejercido por los residentes del lugar, existen garantías de una seguridad más amplia, ya que se instituye una suerte de vigilia colectiva sobre las calles residenciales.

En conclusión, la concurrencia de personas en los espacios públicos es una condición necesaria aunque no suficiente para dar garantías de seguridad. Para que ello ocurra, es necesario que las personas que transitan y permanecen en los espacios públicos conformen un colectivo social, es decir, establezcan lazos comunitarios de vecindad (residan en el lugar).

De allí la importancia clave de la función residencial en los Centros Históricos de las ciudades.

En el Centro Histórico de León se advierte un decremento progresivo de la población del $18 \%$ en el término de 10 años (1990 / 2000), en relación a un aumento del $30 \%$ para todo León en igual período. Esta situación pone de manifiesto una suerte de gentrificación que presiona sobre los habitantes y el usuario de la zona central, a favor de las nuevas poblaciones flotantes de turistas. Sobre esta problemática se expondrá más adelante.

Toda política de renovación urbana debe desarrollarse en conformidad con una política de vivienda (Borja, 2011).

\section{LAS LÓGICAS ESPACIALES DEL DELITO}

\section{Tipificación del delito}

Según sea el objetivo del delito, distinguiremos el delito contra la persona y el delito patrimonial. El primer caso comprende categorías tales como hurto, robo, fraude, etc. El segundo, de mayor impacto social, comprende todos aquellos actos delictivos en contra de la integridad de las personas (homicidio y lesiones), de su salud (delitos relacionados con drogas), en contra de su libertad (secuestro) y el normal desarrollo psicosexual (violación, abuso sexual).

Los mapas M2 y M3 siguientes, dan cuenta del patrón espacial delictivo enn la ciudad de León, según sea contra la propiedad o contra la persona. 

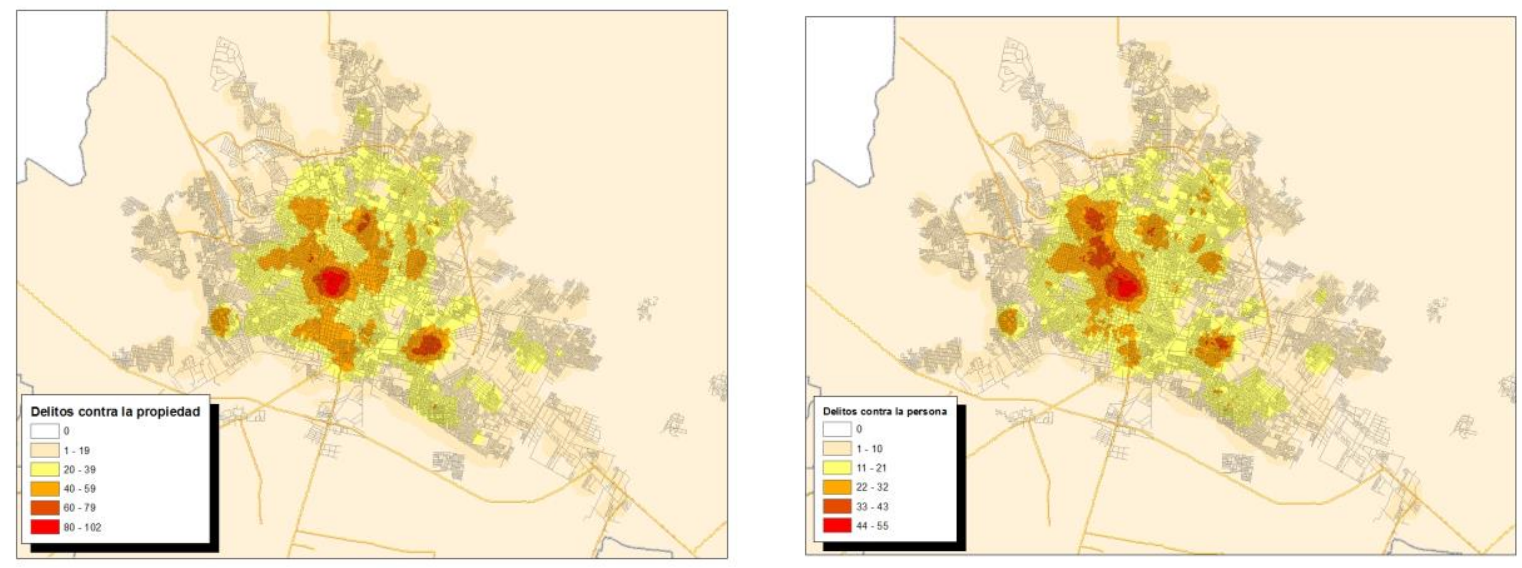

Fuentes: elaboración del Ing. Marco Antonio Lira (IPLANEG), a partir de bases de datos oficiales.

El patrón de comportamiento del delito contra la propiedad se presenta más extendido y disperso si se lo compara con el delito contra la persona. Este último, se concentra mayoritariamente en la zona central y hacia el norponiente.

\section{Aspectos situacionales}

El delito no se manifiesta de forma aleatoria en el espacio o en el tiempo, sino que existen lugares y períodos específicos en los que el delito prevalece (Cohen y Felson, 1979) ${ }^{3}$.

Esto supone calificar la importancia del contexto y las condiciones del ambiente físico (Jeffery, 1971; Newman, 1972) ${ }^{4}$, a diferencia de la disposición criminal del individuo, que se asume constante.

El delincuente se considera un ser relativamente racional que toma decisiones sobre su comportamiento, basadas en un análisis de los daños y beneficios del acto delictivo (Cornish y Clarke, 1986) ${ }^{5}$.

\footnotetext{
${ }^{3}$ Ver teorías de las actividades rutinarias.

${ }^{4}$ Ver teorías que consideran la importancia del ambiente físico en la prevención del delito.

${ }^{5}$ Ver teoría de la elección racional.
} 
A este nuevo paradigma se adscriben también la teoría de la policía comunitaria (Trojanowicz, 1998) y la teoría de las ventanas rotas (Kelling y Wilson 1984), las cuales proponen algunas estrategias concretas de prevención del delito. Esta última, establece que los barrios con signos de decadencia y desorden abren sus puertas a las conductas incívicas. Si el desorden y la decadencia no se detienen y continúan sin control, se convierten en poderosas señales que indican a los incívicos que nada le importa a nadie y que nadie vigila, situación que estimula el crimen.

Sobre la base de las consideraciones precedentes, se presentan los datos vinculados a dos aspectos situacionales claves, el lugar de ocurrencia de delitos y la prevalencia horaria de su comisión.

Mapa M4 - Delitos por colonias, ciudad de León

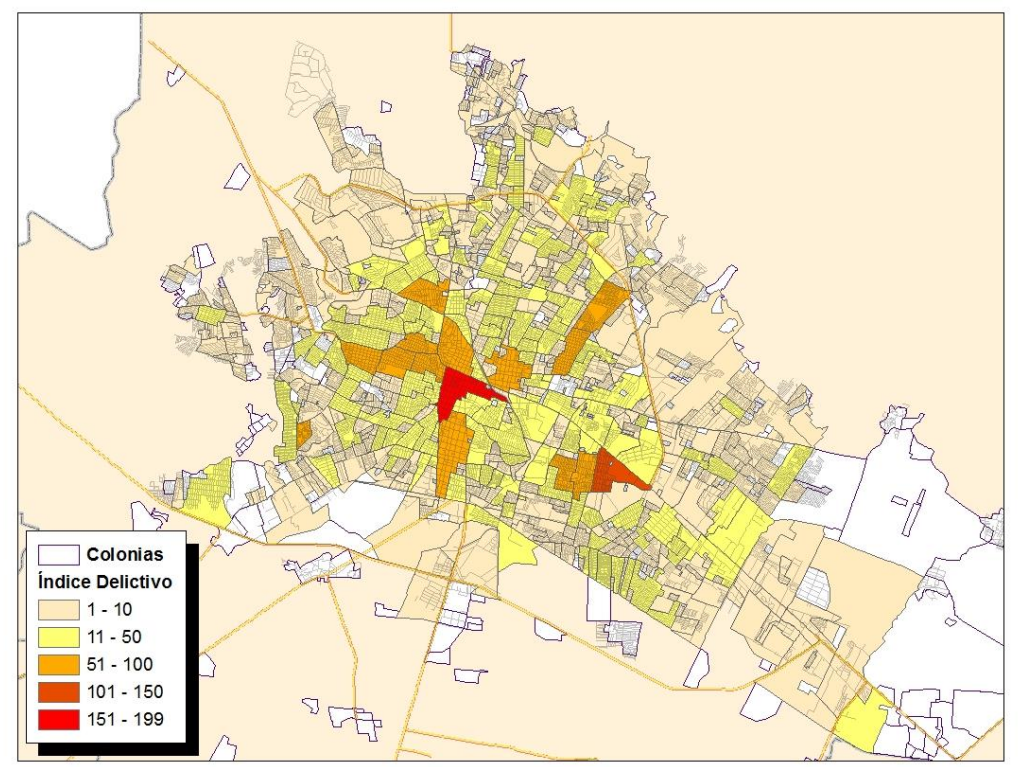

Fuente: elaboración del Ing. Marco Antonio Lira (IPLANEG), a partir de bases de datos oficiales.

Retomando el argumento del colectivo social, resulta imperativo observar la intensidad del problema delictivo en cada una de las colonias del Municipio de León (mapa M4). Ello permite identificar aquellas colonias de mayor incidencia delictiva. Tal es el caso de Las Hilamas y Haciendas de León. Le siguen, en orden decreciente, la colonia Centro, Obregón, San Isidro, La Florida, entre otras. 
Mapa M5 - Variación horaria de la comisión delictiva y su distribución territorial, ciudad de León

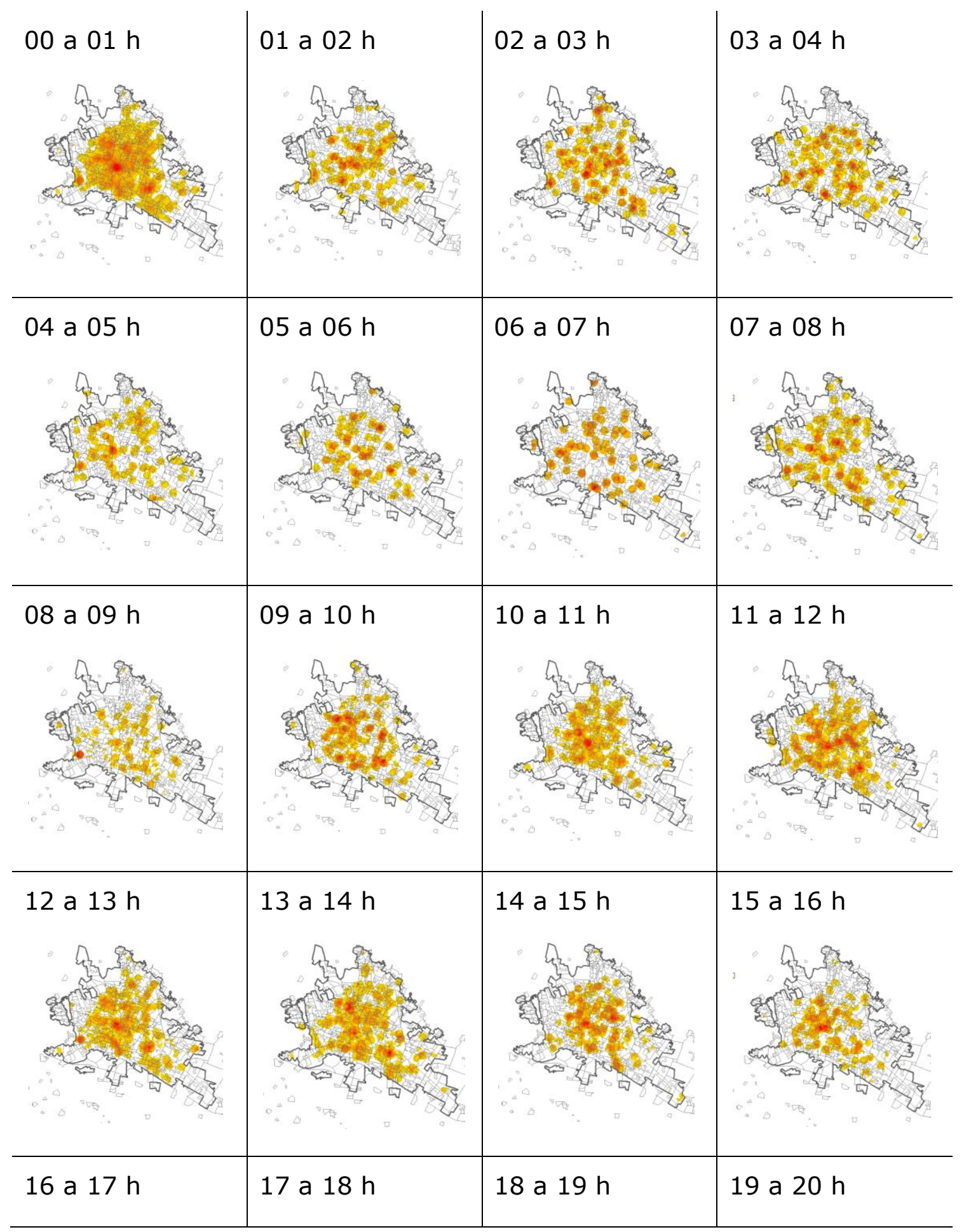




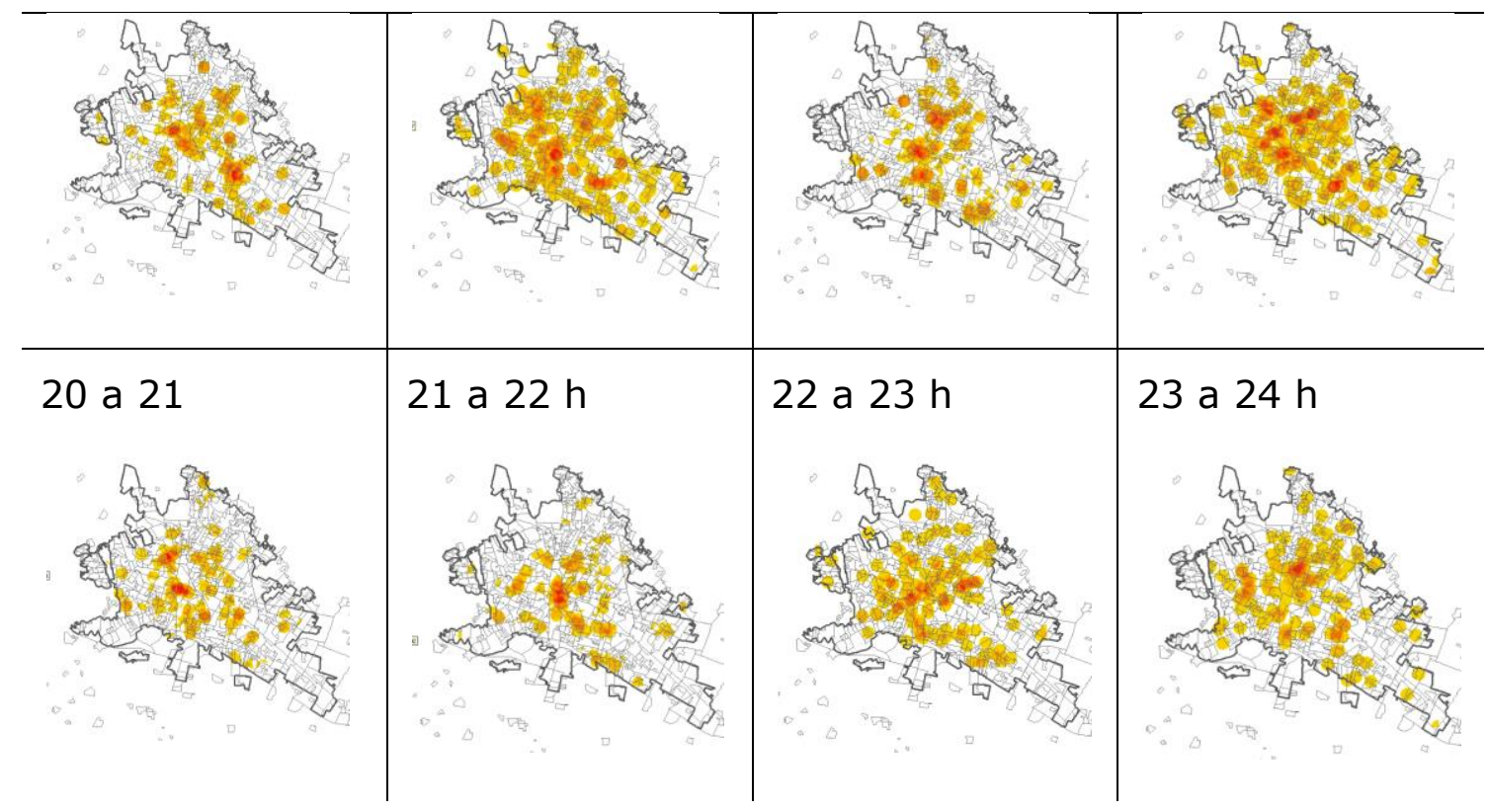

Fuente: elaboración propia, a partir de bases de datos oficiales.

La serie de horarios consignados en el Mapa M5 ilustra la densidad y el comportamiento espacial del delito en cada hora del día solar. Esta secuencia permite identificar, en primer lugar, los rangos horarios críticos de comisión delictiva $y$, en segundo lugar, la mayor o menor concentración en determinadas áreas de la mancha urbana.

De la observación de la información georeferenciada expuesta resulta que la densidad crece y se dispersa territorialmente entre las 7:00 y 8:00 hrs. de la mañana, en coincidencia con la entrada al colegio y el inicio de la jornada laboral en instituciones de gobierno y privadas. Disminuye de 8:00 a 9:00 hrs. para volver ascender a partir de las 10:00 hrs., concentrándose en la zona central de la ciudad. Esta situación permanece más o menos estable hasta las 14:00 hrs., en que acusa una disminución (cierre de comercio). Los períodos comprendidos entre las 17:00 y 18:00 hrs., y las 19:00 y 20:00 hrs. marcan períodos de inflexión ascendentes. En tal sentido, se podría especular con el inicio y cierre de comercios y servicios en horario vespertino, habida cuenta de su concentración en la zona central. Por último, la medianoche representa un horario crítico, con un patrón espacial disperso.

\section{Polígonos de delincuencia}

Empeñados en la tarea de identificar criterios de delimitación de polígonos de delincuencia para la ciudad de León, se ha intentado hacer aproximaciones gráficas a partir de la consideración de dos aspectos: a) que la colonia registre una 
incidencia igual o mayor a 35 hechos delictivos y b) que las colonias sean colindantes.

Esta primera delimitación de áreas (mapa M6) permite explorar indicadores urbanos, ambientales, sociales y económicos más o menos compartidos por las colonias integrantes de cada polígono, con el fin de especular en relación a las causas que abonan la delincuencia.

Mapa M6 - Polígonos de delincuencia, ciudad de León

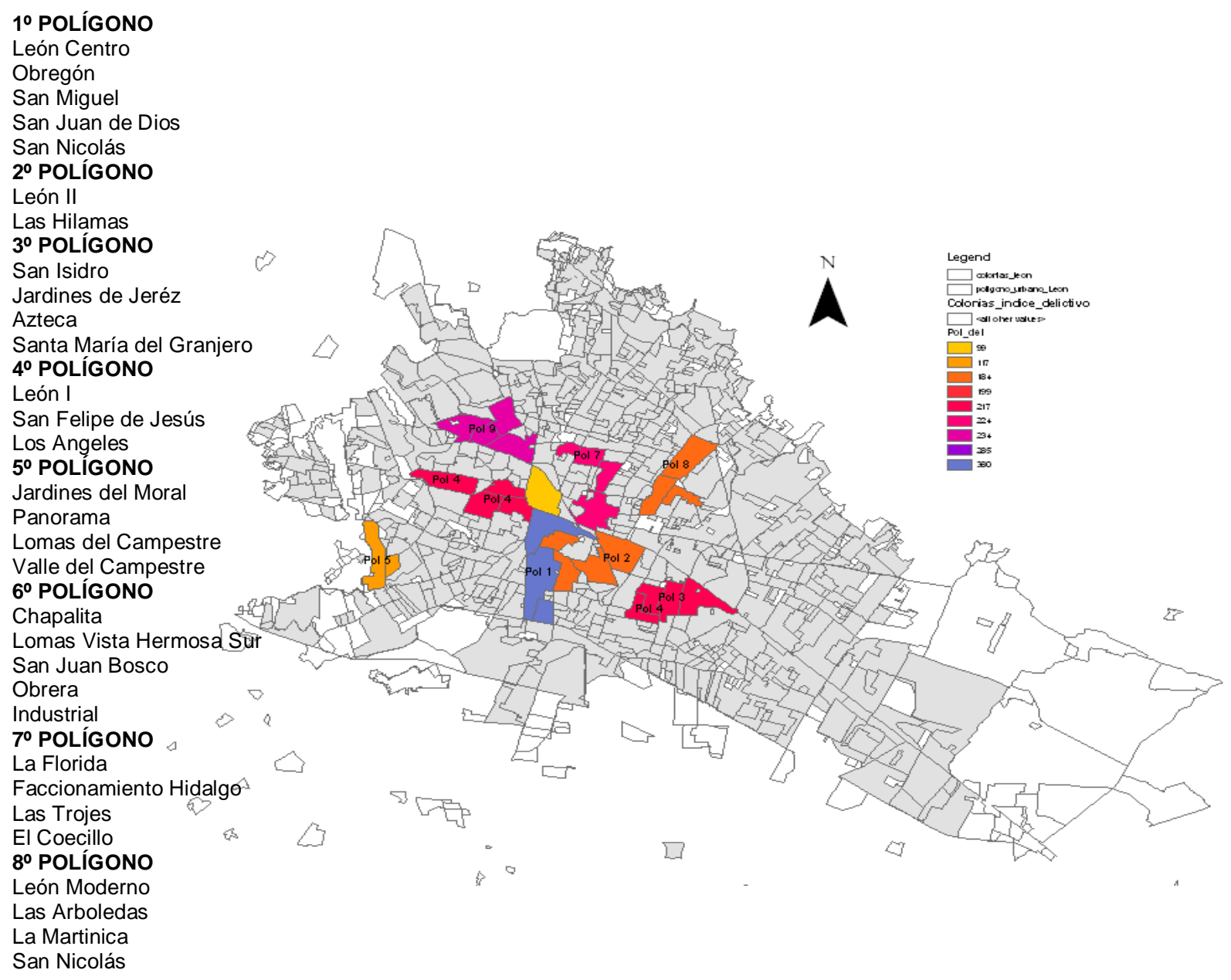

Fuente: elaboración propia, a partir de las bases de datos oficiales. 


\section{Delincuencia y pobreza}

La concentración espacial de la pobreza -polígonos de pobreza- se asocia generalmente a la concentración de desventajas sociales, tales como: bajos ingresos, rezago educativo, carencia de recursos y servicios públicos, vivienda precaria, entre otros, sin mencionar el problema del desempleo y la disrupción familiar. Algunos autores asocian el concepto desventajas sociales al de desorganización social y con ello, destacan el deterioro de los vínculos sociales que impide a estas poblaciones organizarse en comunidad. En tal sentido, las desventajas sociales sumadas a la desorganización social implicarían altos índices de criminalidad. Sin embargo, estas afirmaciones no parecen validarse en cualquier contexto cultural. Tal es el caso de América Latina, donde los procesos de urbanización asumen ciertas particularidades.

En Latinoamérica existen factores endógenos propios de la región que inciden en que los procesos de urbanización no fueron acompañados por idénticos procesos de industrialización. El concepto de "modernización", en tanto desarrollo económico, en América Latina, está asociado al concepto de subdesarrollo (Germani, 1969). La intensa migración del campo a la ciudad desbordó las posibilidades de absorción de mano de obra por parte del mercado de trabajo urbano, produciendo recesión, escasez de recursos y pobreza creciente. Los nuevos asentamientos de la periferia urbana dependían mutuamente, uno del otro, para poder sobrevivir, construyendo redes sociales densas al interior de estas comunidades desposeídas. Estudios recientes en América Latina, demuestran que la cohesión social y la eficacia colectiva están positivamente asociadas a los conceptos de desventaja, desorden y violencia.

En el mismo sentido, Luhmann afirma que, la carencia de recursos hace de la confianza un bien preciado, que disminuye el nivel de complejidad de la vida cotidiana (Luhmann, 1973).

Mapa M7 - Densidad delictiva, ciudad de León y polígonos de pobreza 


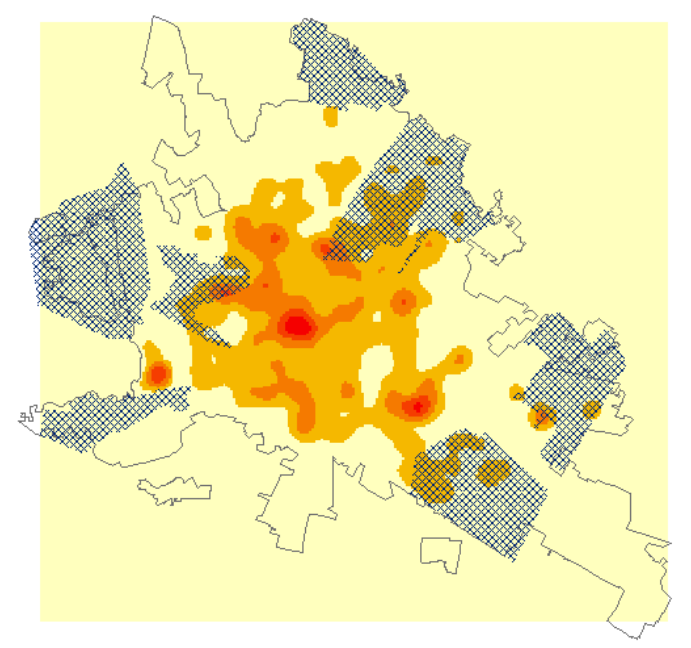

Fuente: elaboración propia, a partir de las bases de datos oficiales.

Mapa M8 - Polígonos de pobreza, ciudad de León

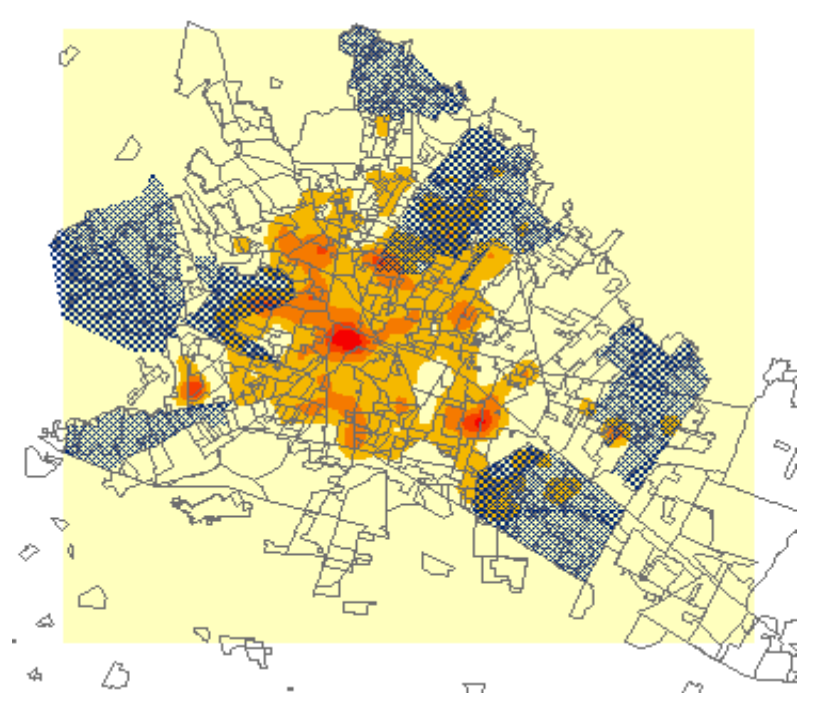

Fuente: elaboración propia, a partir de las bases de datos oficiales.

Teniendo en cuenta las consideraciones previas retomamos nuestra unidad de estudio. 
En los mapas M7 y M8 están representados, además de la densidad delictiva, los siete polígonos de pobreza propuestos por el Instituto Municipal de Planeación de León (IMPLAN). Dicha delimitación especial tiene como antecedentes los criterios de medición establecidos por la Secretaría de Desarrollo Social (SEDESOL) para la determinación de la pobreza. Los indicadores considerados en este caso fueron: a) ingreso corriente per cápita, b) rezago educativo promedio en el hogar, c) acceso a servicios de salud, d) acceso a la seguridad social, calidad de espacios en la vivienda, e) acceso a servicios básicos en la vivienda, g) acceso a la alimentación y, h) grado de cohesión social.

Los resultados espaciales son elocuentes: la causa de los hechos delictivos no parece estar en los bajos índices de pobreza.

Lejos de estigmatizar, con la delincuencia, a las poblaciones con desventajas sociales, llama la atención los bajos índices, cuando no ausencia de delitos en algunos de estos siete polígonos, como es el caso de Los Castillos, San Francisco, La Joyas y Jacinto López.

Dichos resultados pueden tener una explicación en dos sentidos diferentes.

El primero, conduce a la presunción de que tanto víctimas como delincuentes viven en la misma área donde ocurrió el delito, lo cual puede llevar a la confusión. Por ejemplo, la teoría del patrón delictivo sostiene que los delincuentes buscan o encuentran oportunidades a medida que viajan entre las áreas donde realizan la mayoría de sus actividades. Estas áreas incluyen sus lugares de residencia; sin embargo, sus acciones no están exclusivamente limitadas a estos espacios.

El segundo argumento, nos enfrenta a la cifra negra, es decir, al valor poco significativo atribuido a las muestras de los datos oficiales. De acuerdo a los resultados de la investigación que precede a la presente, se estima que la cifra negra en León asciende al 80\%. A favor de este argumento, cabría esperar que estas poblaciones tengan poca o nula cultura de denuncia, ya que también se ha comprobado que la población victimizada se manifiesta reticente a denunciar los delitos, por razones que oscilan desde la desconfianza en la justicia hasta considerarlo una pérdida de tiempo, pasando por restarle importancia al hecho.

Dimensiones tales como la densidad poblacional y el nivel de población masculina entre 15 y 26 años, importan para ser vinculadas a la delincuencia, habida cuenta de los altos índices de pandillerismo. En el territorio de la ciudad de León se registran 797 pandillas activas. La presencia de jóvenes pandilleros se asocia al desorden social, el cual impacta negativamente en la acción colectiva de su comunidad de pertenencia. 


\section{Delincuencia y control policial}

Resulta interesante analizar la distribución en la mancha urbana de los Centros de Seguridad y su relación con la delincuencia. El mapa M9 da cuenta de la localización del equipamiento vinculado a la Seguridad con un radio de cobertura de 500 metros, a la vez que presenta la densidad de hechos delictivos.

Mapa M9 - Delincuencia y Centros de Seguridad

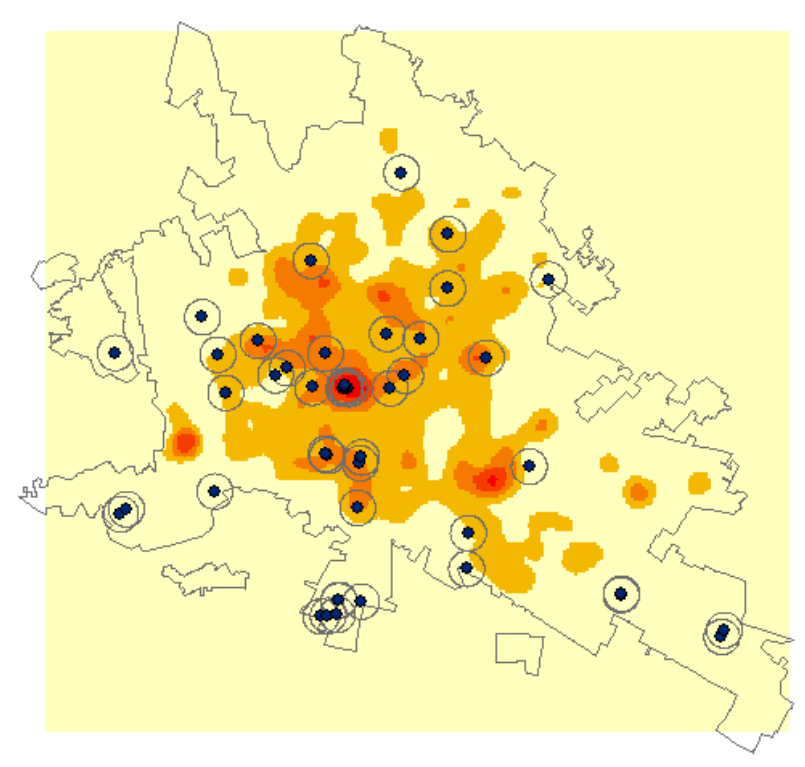

Fuente: elaboración propia, a partir de las bases de datos oficiales.

El control policial opera en la dirección opuesta a la esperada, mostrando una predicción positiva en las tasas delictivas. Una explicación de esta relación puede ser que los recursos destinados a estas unidades son distribuidos en forma reactiva y no proactiva. Esto quiere decir que tanto las entidades públicas como los residentes buscan garantizar la inversión de recursos en las áreas que presentan los mayores índices de delincuencia. Este puede ser el caso del Centro Histórico de la ciudad de León, donde se concentra un número significativo de controles policiales.

\section{CONCLUSIÓN}


No existen dudas del valor instrumental de la geografía en las tareas de diagnóstico. El caso que nos ocupa, la violencia urbana, se manifiesta de manera solidaria con las condiciones de contexto y en tal sentido, su carácter es voluble e imprevisible.

En consecuencia, la validez de su diagnóstico reside en una permanente actualización de las fuentes de información, que permita llevar a cabo su traducción espacial en forma inmediata. De lo contrario, los esfuerzos por medir y cualificar el fenómeno serán siempre desbordados por las cambiantes lógicas de su comportamiento.

\section{REFERENCIAS}

Akerman, M. (1997). Mapa de risco da violência da cidade de São Paulo: explorando os diferenciais intra-urbanos. Presentado en Seminario sobre violencia criminal urbana. Rio de Janeiro: Banco Interamericano de Desarrollo (BID).

Ascher, F. (2004). Los nuevos principios del urbanismo. Ensayo. Madrid: Alianza.

Beck, U. (1999). World risk society. Cambridge: Polity Press.

Borja J. (2011). Urbanismo Socialmente Responsable. Ponencia presentada en el III Seminario Internacional de urbanismo Socialmente Responsable. Guanajuato, México: IMPLAN León.

Carrión F, Cobo G. (1994). (Eds.) Ciudad y violencias en América Latina. Quito: Programa de Gestión Urbana.

Cohen, L. E. y Felson, M. (1979). Social change and crime rate trends: A routine activity approach. En American Sociological Review, 44, 588-608.

Cornish, D. y Clarke, R. V. (1986). (Coord.) The Reasoning Criminal. Nueva York: Springer-Verlag.

Germani, G. (1969). La sociología de la modernización. Buenos Aires.

Jacobs, J. (1901). Muerte y vida de las grandes ciudades. Madrid: Península.

Jeffery, C. R. (1971). Crime Prevention Through Environmental Design. Beverly Hills: Sage.

Kelling, G. \& Wilson, J. Q. (1984). Broken Windows: The police and neighborhood safety. En The Atlantic Monthly.

Luhmann, N. (1979)., trad. esp. 1996:14), Confianza, Anthropos, México 
Newman, O. (1972). Defensible Space: Crime Prevention through Urban Design. Nueva York: Macmillan.

Trojanowicz, R. y Bucqueroux, B. (1998). Community Policing: How to get started. Ohio: Anderson.

Vanderschueren F. (1994). La violencia urbana, los pobres de la ciudad y la justicia. En Concha Eastman A., Carrión F. \& Cobo G. (Eds.) Ciudad y violencias en América Latina, pp. 5 - 22. Quito: Programa de Gestión Urbana.

Proyecto IDEAL, Programa URB-AL III de la Comisión Europea. Una nueva estrategia de Gobernanza Local. La intermunicipalidad en América Latina. México: Gobierno del Estado de Michoacán de Ocampo; Uruguay: CLAEH. 2012.

\section{Alicia Ziccardi ${ }^{6}$}

Este importante documento de trabajo puede contribuir en mucho a perfeccionar los procesos de gestión de los municipios latinoamericanos, aquellos que quieren lograr mejores condiciones de vida para su ciudadanía. En este sentido, el Proyecto IDEAL (Una nueva estrategia de Gobernanza local. La Intermunicipalidad en Europa y América Latina), ofrece un título muy sugerente, porque ciertamente el proyecto tiene algo o mucho de ideal, de utopía; ha sido iniciado hace cinco años y busca promover nuevas formas de gobernanza del territorio local, creando una moderna estrategia de actuación compartida.

Se trata de un proyecto que forma parte del Programa URBAL III, de la Comisión Europea, una asociación de gobiernos locales de siete países, cinco de los cuales son latinoamericanos y dos, europeos.

Es un libro con un gran valor pedagógico y que, a mi entender, tiene dos importantes méritos:

1) Aporta ideas a quienes quieren innovar y democratizar la gestión local. Más específicamente, contribuye a crear una nueva forma de gestión de las políticas

${ }^{6}$ Completar información bibliográfica de la autora de la Reseña. PUEC UNAM. 